\title{
Effects of ECRH power and safety factor on laser blow-off injected impurity confinement in TCV
}

\author{
E. Scavino, J. Bakos ${ }^{+}$, H. Weisen and TCV Team \\ Centre de Recherches en Physique des Plasmas, \\ Association EURATOM - Confédération Suisse, \\ Ecole Polytechnique Fédérale de Lausanne EPFL \\ CH-1015 Lausanne, Switzerland \\ ${ }^{+}$KFKI, Budapest, Hungary
}

Short title: Impurity confinement in TCV

PACS: 52.25.Vy

\begin{abstract}
Evidence from the injection into the TCV device of laser ablated, non-recycling silicon impurities, shows that the transport of impurities confinement can be remarkably different from that of energy. The ratio of impurity to energy confinement times ranges from near unity in Ohmic discharges to 5 in the presence of high power ECCD. In Ohmic discharges in Deuterium, above a threshold of density and of safety factor near $q_{95}=4.5$, the impurity confinement time increases abruptly by a factor of 2 and is sometimes accompanied by indefinite retention of non-recycling impurities within the sawtooth mixing radius, despite the continued presence of sawteeth.
\end{abstract}

\section{Introduction}

The control of the production and transport of impurities is of vital interest for future fusion power generating devices. Impurities affect the power balance by radiation losses and fuel dilution. At the same time impurities radiating a substantial fraction of the plasma loss power near the edge may prove necessary to maintain power densities on the heat load components at tolerable levels [1]. The controlled injection of impurities can even improve energy confinement [2]. Despite this important role, impurity transport is often neglected and given little attention and low priority in the preparation of experimental programmes aimed at investigating transport in fusion experiments. The same can be said of some of the theoretical 
work on transport. While impurities were given due attention in the development of neoclassical theory $[3,4]$, they have so far received little attention from theorists modelling anomalous transport, which is responsible for the lions share of transport over most of the plasma cross section. The results presented, together with those previously published on the dependence of impurity transport on plasma shape [5,6] show a bewildering range of behaviour and demonstrate that some modelling assumptions on the relationship between impurity and energy transport coefficients are untenable. It is hoped that observations such as these will encourage a concerted effort between experimentalists and theorists for systematic and simultaneous investigations of the transport properties of all plasma constituents.

The TCV tokamak is a versatile device, which allows the production of a wide range of discharge conditions [7]. Extensive investigations were carried out on the effects of the plasma shape both on heat transport [8,9] and on impurity transport [5]. The energy confinement time was observed to decrease both with increasing plasma current [9] and with increasing ECRH power [10] in agreement with the scaling behaviour observed in other tokamak devices. The experiments reported in section 2.1 and 2.2 below were aimed at investigating whether the additional heating power and the current (or safety factor) have analogous effects on the confinement of impurities. A phenomenon of impurity accumulation observed in some discharges, leading to apparently indefinite retention of impurities in the core, is described in section 2.3.

\section{Experiments}

The TCV tokamak $\left(R_{0}=0.88 \mathrm{~m}, a<0.25 \mathrm{~m}, B_{T}<1.5 \mathrm{~T}, 1<\kappa<2.8,-0.8<\delta<0.9\right)$ [1] allows the production of plasma currents up to $1 \mathrm{MA}$ and it is equipped with 6 gyrotrons for ECRH and ECCD operating at the second electron cyclotron harmonic frequency, with a total power of 2.8 MW available at the launchers for heating the plasma [11]. The standard laser blow-off technique[12,13] was used to inject trace (i.e. non perturbing) quantities of the nonrecycling element silicon. Injections were performed during the current plateau, at a rate of 1 per plasma discharge using a single pulse ruby laser.

A 200 channel soft x-ray (SXR) photodiode array with $47 \mu \mathrm{m}$ thick Be filters served as the main diagnostic tool for this study [14]. The spectral sensitivity of the filtered photodiodes ( $>0.5$ for photons in the range $2.5-11 \mathrm{keV}$ ) is well suited for the detection of line emission and 
continuum radiation from highly ionised states of moderate- $\mathrm{Z}$ impurities in the core of TCV plasmas. The cameras, whose lines of sight fully cover a single poloidal plane, allow a resolution of $30-40 \mathrm{~mm}$ after performing a tomographic reconstruction of the local SXR emissivity[14]. The acquisition system has a maximum sampling rate of $80 \mathrm{kHz}$, which is much faster than the typical transport timescales of impurities in TCV [5].

The general features and the analysis technique of the propagation of impurities in TCV plasmas have been described in Ref.[5]. The global impurity confinement time $\tau_{\text {imp }}$ is defined as the time constant of the exponential decay of the line integrated SXR signals, following the peak of the pulse produced by the introduction of the impurities into the plasma. This value is systematically observed to be the same for all viewing lines, with the notable exception of discharges which indefinitely retain impurities in the plasma core. Prior to fitting a decreasing exponential, sawteeth are removed from the SXR signals using the GSVD method [15] for enhanced accuracy. Unless otherwise indicated, the discharges discussed in the following are sawtoothing L-mode deuterium plasmas.

\subsection{ECRH power dependence}

A scan of ECRH power was performed in a series of 9 consecutive limiter discharges, with a maximum additional power of $1 \mathrm{MW}$ from two gyrotrons. The other parameters were kept constant with elongation $\kappa_{a}=1.6$, triangularity $\delta_{a}=0.33$, plasma current $I_{P}=260 \mathrm{kA}$, line integrated electron density $\bar{n}_{e}=1.5 \times 10^{19} \mathrm{~m}^{-3}$ and toroidal magnetic field $B_{T}=1.47 \mathrm{~T}$. The central electron temperature rose from near $1.1 \mathrm{keV}$ with Ohmic heating only to $2.5 \mathrm{keV}$ with $1 \mathrm{MW}$ of ECRH.

The effects of the ECRH power scan on both the impurity and the total energy confinement times are reported in Fig.1. The total energy confinement time is based on a measurement of stored energy using a diamagnetic loop. Both confinement times are seen to decrease with increasing heating power. The phenomenon, known as power degradation is ubiquitous in fusion plasmas [16]. The effect is however less pronounced for the impurities. The energy confinement in this experiment varies as $\tau_{E} \propto P_{\text {tot }}^{-0.4}$, while the impurity confinement varies only as $\tau_{i m p} \propto P_{t o t}^{-0.2}$, as indicated by the power law curves fitted in Fig.1.

Different experiments with larger auxiliary power, although not systematic, have yielded ratios of impurity to energy confinement time up to 5 and is listed for two examples with full EC current drive (zero loop voltage) in table 1. 


\begin{tabular}{|c|c|c|c|c|c|c|c|c|}
\hline Shot \# & $\mathrm{P}_{\mathrm{FCCD}}[\mathrm{MW}]$ & $\tau_{\mathrm{imn}}[\mathrm{ms}]$ & $\tau_{\mathrm{F}}[\mathrm{ms}]$ & $\mathrm{I}_{\mathrm{n}}[\mathrm{kA}]$ & $\left\langle\mathrm{n}_{\mathrm{e}}\right\rangle\left[10^{19} \mathrm{~m}^{-3}\right]$ & $\kappa$ & $\delta$ & $\mathrm{T}_{\mathrm{e}}(0)[\mathrm{keV}]$ \\
\hline 19485 & 1.35 & 16 & 4.2 & 135 & 1.2 & 1.47 & 0.52 & 3.5 \\
19557 & 1.8 & 10 & 2.1 & 120 & 0.8 & 1.35 & 0.33 & 4 \\
\hline
\end{tabular}

Table 1: Energy and impurity confinement times in high power ECCD discharges.

It may be of importance to note that ions in these discharges are only heated by collisions with electrons. At the densities of the above experiments ions are decoupled and remain at temperatures of a few hundred $\mathrm{eV}$ (a small fraction of the electron temperature), as measured in similar discharges using Charge Exchange Spectroscopy [17]. In these conditions the ion thermal energy represents less than $20 \%$ of the total plasma thermal energy. A tentative explanation for the weak power dependence of the impurity confinement time may be that the impurity confinement is related to that of the main ions (deuterium and carbon). Since these are decoupled from the electrons, they receive little heating and may hence experience little power degradation. A similar behaviour has been reported from Tore Supra, $\tau_{p} \propto P_{t o t}^{-0.2}$, for the main ions and was deduced from electron density and particle flux measurements to the limiters [18]. By contrast a joint investigation of high Z (Manganese, Nickel, Copper, Germanium) impurity confinement in JET and Tore Supra L-modes plasmas produced a common scaling law with strong power degradation, $\tau_{z} \propto P_{t o t}^{-0.57}$, similar to that of the plasma energy confinement [19]. Remarkably, in the JET-Tore Supra studies, the total energy confinement time exceeded the impurity energy confinement time by a factor of 2.5 on average, whilst for TCV experiments, as reported here and previously [5], the impurity confinement time is the larger with $1 \leq \tau_{z} / \tau_{E}<5$.

\subsection{Safety factor dependence in Ohmically heated discharges}

The scan of plasma current $I_{P}$ was performed with a series of 11 consecutive limiter discharges, covering the range $I_{P}=163 \div 525 \mathrm{kA}$, with Ohmic heating only. The associated variation of the safety factor at $95 \%$ of the minor radius was $q_{95}=6.4 \div 2.2$ and of the sawtooth inversion radius was $\rho_{i n v}=0.2 \div 0.55$, since the geometry and the toroidal magnetic field were kept constant for all discharges. The other parameters were $\kappa_{a}=1.6$, $\delta_{a}=0.28, B_{T}=1.47 \mathrm{~T}$ and $\bar{n}_{e}=4.0 \times 10^{19} \mathrm{~m}^{-3}$, line averaged.

The results of the scan are shown in Fig.2. A sharp threshold appears at $I_{P} \approx 250 \mathrm{kA}$, corresponding to $q_{95} \approx 4.5$, above which the impurity confinement times $\tau_{\text {imp }}$ are around the 
typical value of $25 \mathrm{~ms}$, as observed in previous experiments [5]. Agreement with the observations on other tokamaks[20,21] is seen in the range $I_{P}=250 \div 480 \mathrm{kA}$ where the impurity confinement time shows a slight increase with the current. Below the threshold at $I_{P} \approx 250 \mathrm{kA}$ the impurity lifetime increases abruptly to values above $50 \mathrm{~ms}$, with some of the discharges, described in the next section, retaining impurities indefinitely in their core.

Unfortunately, at the time this experiment was performed, a diamagnetic loop was not yet available. It was also not possible to evaluate the electron energy confinement time because of the absence of Thomson scattering measurements on most of the discharges. Fortunately however, the scaling of the energy confinement time with plasma parameters in TCV is well documented from previous investigations. For Ohmic plasmas a version of the Neo-Alcator law for the total energy confinement time, modified for including the effect of plasma shaping [9], is applicable. It can be expressed as $\tau_{N A-T C V}=1.15 \times 10^{-21} H_{S}\left\langle n_{e}\right\rangle a R_{0}^{2} q_{\text {eng }}$, where $q_{\text {eng }}=2 \kappa a B_{T} /\left(\mu_{0} R_{0} I_{p}\right)$ and $\mathrm{H}_{\mathrm{s}}$ is a factor close to unity, describing the effect of shaping. All other parameters of the series being constant, the energy confinement time decreases continuously with increasing current. Although there is a factor-of-two reduction of $\tau_{i m p}$ with $I_{P}$ over the range of the scan, the observed threshold behaviour cannot be related to the behaviour of $\tau_{E}$.

The 1-D simulation code STRAHL[22, 23] has been used to estimate the transport parameters $D_{\text {edge }}$ and $v_{\text {edge }}$ of the impurities for two injections in plasmas whose currents were respectively below and above the threshold. The simulation technique is identical to that described in Refs.[6, 7] and similarly the radial profiles of the transport parameters were chosen to be parabolic, $D(\rho)=D_{0}+\left(D_{\text {edge }}-D_{0}\right) \rho^{2}$, for the diffusivity and linear, $v(\rho)=v_{\text {edge }} \rho$, for the convective velocity. The range of best fitting values, reported in Table 2 , indicate clearly that a change in the direction of the convective velocity is associated to the factor-of-two increase in the impurity confinement time below the current threshold at $I_{P} \approx 250 \mathrm{kA}$. The value of $v_{\text {edge }}$ for the injection at $I_{P}=350 \mathrm{kA}$ is in agreement with the results reported in Ref.[5], where the edge velocity was always observed to be outwardly directed. For these injections, the central diffusivity $D_{0}$ could not be determined with sufficient precision, as in the simulations described in Ref.[5]. 


\begin{tabular}{|c|c|c|c|c|}
\hline Shot \# & $\boldsymbol{I}_{P}[\mathbf{k A}]$ & $\boldsymbol{\tau}_{\text {imp }}[\mathbf{m s}]$ & $\boldsymbol{D}_{\text {edqe }}\left[\mathbf{m}^{2} / \mathbf{s}\right]$ & $\boldsymbol{v}_{\text {edqe }}[\mathbf{m} / \mathbf{s}]$ \\
\hline \hline 20161 & 233 & 55 & $0.64 \div 0.68$ & $-4 \div-3.5$ \\
\hline 20167 & 351 & 25 & $0.84 \div 0.88$ & $3 \div 3.3$ \\
\hline
\end{tabular}

Table 2: Transport parameters of two selected shots in the plasma current scan

The plasma current scan was repeated with a different line integrated electron density, at $\bar{n}_{e}=5.0 \times 10^{19} \mathrm{~m}^{-3}$, and slightly different shape $\left(\kappa_{a}=1.43, \delta_{a}=0.37\right)$. The current threshold was present but occurred for $I_{P} \approx 200 \mathrm{kA}$, corresponding again to $q_{95} \approx 4.5$, as in the previous case. The values of the impurity confinement time $\tau_{\text {imp }}$ were close to those in the previous scan, $\tau_{\text {imp }} \approx 22 \mathrm{~ms}$ for currents above the threshold and $\tau_{\text {imp }} \approx 65 \mathrm{~ms}$ below. When the current scan was repeated in a He background plasma, an continuous overall increase of $\tau_{\text {imp }}$ with $\mathrm{q}_{95}$ was observed, but no sharp threshold.

\section{$\underline{2.3 \text { Persistent impurity accumulation }}$}

A number of injections performed into Deuterium plasmas whose currents, in both scans, are close to the thresholds, reveal a peculiar phenomenon of impurity accumulation in the plasma centre. The line integrated SXR signals from central chords show no or little decrease of the intensity, while the signal from more peripheral chords decays almost linearly to the background. These extremely peaked profiles were observed to last up to the end of the current plateau of the discharge. The behaviour of this accumulation phenomenon is reported in Fig.3 for an injection at $I_{P}=235 \mathrm{kA}$ in the first current scan. The correlation between the position of the chord and the residual signal is evident, and a determination of the global impurity confinement time would be meaningless. The plasma centre appears to indefinitely retain the impurities, while the time evolution of the peripheral signal, although not exponentially decaying, suggests a peripheral confinement time of about $80 \mathrm{~ms}$, in agreement with discharges with $q_{95}>4.5$ presented above, which do not present indefinite accumulation.

Fig.4 shows the normalised residual asymptotic profile of the SXR emissivity due to the injected impurities (after subtraction of the background) obtained by tomographic inversion of the line integrated signals $200 \mathrm{~ms}$ after injection, during the sawtooth build-up phase (solid line). For comparison, the figure also shows the broader profile obtained for $I_{P}=350 k A$ during the exponential decay phase flowing the peak of the SXR pulse (broken line). The peaked profile of impurities can be explained with a very high ratio of the inward 
convective velocity to the particle diffusion coefficient $|v / D|$. In this case, the convective flux appears to be large enough to overcome the diffusive flux due to the density gradient over a large fraction of the plasma cross section. Sawteeth have been filtered out of the SXR traces in Fig.3 using the GSVD method for clarity. It is important however to note that sawteeth persist throughout the accumulative phase as seen in Fig.5, which shows a raw SXR signal with no filtering applied. Sawtooth crashes have the effect of expelling energy and particles from the core, beyond the inversion radius, up to the mixing radius [24]. It is well known that heat deposited beyond the inversion radius is rapidly transported to the edge, giving rise to the characteristic sawtooth heat pulse (see e.g. ref [25]). Impurities in this discharge behave differently. The persistence of impurities in the core after tens of sawtooth crashes shows that the region of strong inward convection must extend at least to the mixing radius. If the safety factor profile is close to parabolic, the mixing radius can be estimated as $\rho_{\text {mix }} \approx \sqrt{2} \times \rho_{\text {inv }}$. From Fig. 3 we see that within a critical radius $0.48<\rho_{\text {crit }}<0.67$, beyond the sawtooth inversion radius $\left(\rho_{\text {inv }} \approx 0.27\right.$ ) and the estimated mixing radius, impurities return to the core after every crash, while beyond this radius, they eventually diffuse to the edge. The reason for the appearance of such a wide region with a strong inward convection is not understood.

A simulation with STRAHL, which was performed to reproduce the profile of the SXR emissivity shown in Fig.4 suggests $v \approx-4 \mathrm{~m} / \mathrm{s}$ at the position of the critical radius $\rho_{\text {crit, }}$ assuming the same diffusion coefficient as for shot 20161 in Table 1 . This value will be an overestimate, if for any reason $\mathrm{D}$ is reduced inside $\rho_{\text {crit, }}$ as compared to non-accumulating discharges.

The behaviour of this discharge is similar to that observed in the TEXTOR tokamak and described as an 'accumulative instability' [26, 27]. In this picture high $\mathrm{Z}$ impurities reaching the plasma core, where anomalous transport is low enough to allow neoclassical transport to compete, can affect the local power balance by increasing radiation losses. The concomitant central cooling can lead to a reduction of the neoclassical screening effect provided by the ion temperature gradient and a reduction of current density and magnetic shear, which may further reduce anomalous transport leaving core transport governed by neoclassical effects. When $\mathrm{q}_{0}$ rises above unity, sawteeth are suppressed, accumulation proceeds further and core radiation rises to higher levels. The case reported here can be seen as one where core radiation remained insufficient to suppress sawteeth, possibly because of the modest amount of Si injected. Accumulation with persisting sawteeth has also been reported from JET Argon-seeding experiments [28]. Accumulative discharges in which sawteeth disappeared, to be replaced by sporadic minor disruptions, as observed in TEXTOR [26], have however been observed in the 
second current scan mentioned under 2.2 above for $\mathrm{q}_{95}>4.5$. An attempt to expel accumulated impurities from the plasma core using central ECH, as demonstrated on ASDEX-UPGRADE [29] proved unfeasible on TCV because at the low densities $\left(\mathrm{n}_{\mathrm{e}}(0)<4 \times 10^{19} \mathrm{~m}^{-3}\right)$ at which the electromagnetic heating waves $(82.7 \mathrm{GHz})$ can propagate to the core, this phenomenon of persistent accumulation was not observed, nor was persistent accumulation observed in $\mathrm{He}$ plasmas.

According to neoclassical theory, inward impurity convection can be prevented by ion temperature screening, provided the background plasma ion collisionality $\mathrm{v}_{\mathrm{b}}{ }_{\mathrm{b}}$ (including main ions and intrinsic impurities) is low enough $v^{*}{ }_{b} \leq 2[3,4]$. For typical values of $Z_{\text {eff }} \sim 2$, the plasma ion collisionality is dominated by intrinsic impurities. At mid-radius the main ion collisionalities have been estimated for the accumulating discharge above as $v_{b}{ }_{b}>10$, as $v_{b}^{*} \sim 5$ in the low density experiments and $v^{*}{ }^{*} \sim 2$ in He plasmas, suggesting that screening may only be effective in He plasmas in TCV. He plasmas have very low levels of intrinsic carbon due to the absence of chemical sputtering. The fact that inward convection is observed at fairly high values of $\mathrm{q}_{95}$, when main ion density profiles are peaked [30], reinforcing neoclassical inward convection [3], is consistent with neoclassical theory. However the fact that only discharges close to the threshold $\left(\mathrm{q}_{95} \cong 4.5\right)$ retain impurities indefinitely remains unexplained. To the best of our knowledge, the observation indefinite trapping of impurities inside the mixing radius of an L-mode discharge, appears to be a novel element in the phenomenology of the accumulative instability. It may also hint towards a mechanism, which could contribute towards explaining the bewildering behaviour of the global residence time in non-accumulating discharges. It is easy to see that the time constant of the decay phase of an LBO pulse would depend sensitively on $\rho_{\text {crit }}$ if $\rho_{\text {invt }}<\rho_{\text {crit }}<\rho_{\text {mix }}$. An experimental verification of this idea would however be a diagnostics challenge.

\section{Conclusions}

The confinement of small amounts of laser-ablated $\mathrm{Si}$ impurities has been investigated in ECH and ECCD plasmas and as a function of plasma current in Ohmic plasmas. These investigations, together with those previously reported on the effect of plasma shaping [5], show that the global energy confinement time is a poor predictor for the impurity confinement time. In TCV conditions the impurity confinement remains little affected by power degradation, $\tau_{\text {imp }} \propto P_{\text {tot }}^{-0.2}$, whereas the electron energy confinement varies as $\tau_{E} \propto P_{\text {tot }}^{-0.4}$. In high 
power ECH and ECCD discharges, when electrons and ions are decoupled, the ratio of impurity to energy confinement time can be as high as 5 .

The scan of plasma current has yielded a surprising threshold in the behaviour of $\tau_{\text {imp }}$, as well as phenomena of impurity accumulation in the plasma centre. While for $\mathrm{q}_{95}<4.5$, there is little effect on the confinement time of impurities, for higher values $\tau_{i m p}$ increases twofold. Transport simulations with the code STRAHL indicate that the threshold corresponds to a reversal of the convective velocity from outward to inward. A phenomenon of persistent impurity accumulation similar to the accumulative instability in TEXTOR [26,27] was observed for $\mathrm{q}_{95}$ just above the safety factor threshold at central densities above $4 \times 10^{19} \mathrm{~m}^{-3}$. In the case reported, sawteeth persist until the end of the discharge and impurities remain trapped indefinitely within the mixing radius, whilst impurities outside the mixing radius diffuse to the edge and leave the plasma.

\section{Acknowledgements}

This work was partly supported by the Swiss National Science Foundation.

\section{References}

1. ITER Physics Expert Groups, ITER Physics Basis, Nuclear Fusion 39 (1999) 2391

2. M.Z. Tokar et al, Phys. Rev. Lett. 84 (2000) 895

3. S.P Hirshman, D.J. Sigmar, Nuclear Fusion 9 (1981) 1079

4. A.G. Peeters, Phys. Plasmas 7 (2000) 268

5. E.Scavino et al., Plasma Phys. Contr. Fus. 45 (2003), 1961

6. E.Scavino, PhD Thesis No. 2677, Ecole Polytechnique Fédérale de Lausanne, Switzerland (2002); CRPP-EPFL report LRP 758/03 (2003)

7. F.Hofmann et al., Plasma Phys. Contr. Fusion 36 (1994), B277

8. J.M.Moret et al., Phys. Rev. Lett. 79 (1997), 2057

9. H.Weisen et al., Plasma Phys. Control. Fusion 39 (1997), B135

10. A. Pochelon et al, Nuclear Fusion 39 (1999) 1807

11. T.P.Goodman et al., Proc. $19^{\text {th }}$ SOFT, Lisbon (1996), 565

12. J.F.Friichtenicht, Rev. Sci. Instr. 45 (1974), 51

13. E.S.Marmar et al., Rev. Sci. Instr. 46 (1975), 1149

14. M.Anton et al., Plasma Phys. Control. Fusion 38 (1996), 1849

15. T. Dudok de Wit et al., Phys. Plasmas 5 (1998) 1360

16. ITER Physics Expert Groups, ITER Physics Basis, Nuclear Fusion 39 (1999) 2175

17. P. Bosshard, CRPP-EPFL report LRP 750/03, (2003) under preparation for publication

18. B. Pégourié et al., J. Nuclear Materials 241-243 (1997) 494

19. M. Mattioli et al., Nuclear Fusion 35 (1995) 1115

20. K.W.Gentle et al., Plasma Phys. Control. Fusion 26 (1984), 1407

21. S.Fairfax et al., Nucl. Fusion Supplement 1 (1981), 439

22. K.Behringer, Jet report JET-R(87) 08 (1987) 
23. R.Dux, STRAHL User Manual (2000)

24. F. Porcelli et al, Nuclear Fusion 40 (2000) 1691

25. N.J. Lopes Cardozo and A.C.C Sips, Plasma Phys. Control. Fusion 33 (1991) 1337

26. J. Rapp et al., Plasma Phys. Control. Fusion 39 (1997) 1615

27. M.Z. Tokar et al, Plasma Phys. Control. Fusion 37 (1995) A241

28. M E Puiatti et al. Plasma Phys. Control. Fusion 45 (2003) 2011

29. R. Dux et al., J. Nuclear Materials 313-316 (2003) 1150

30. H. Weisen et al., Plasma Phys. Control. Fusion 40 (1997) 1803 


\section{Figures}

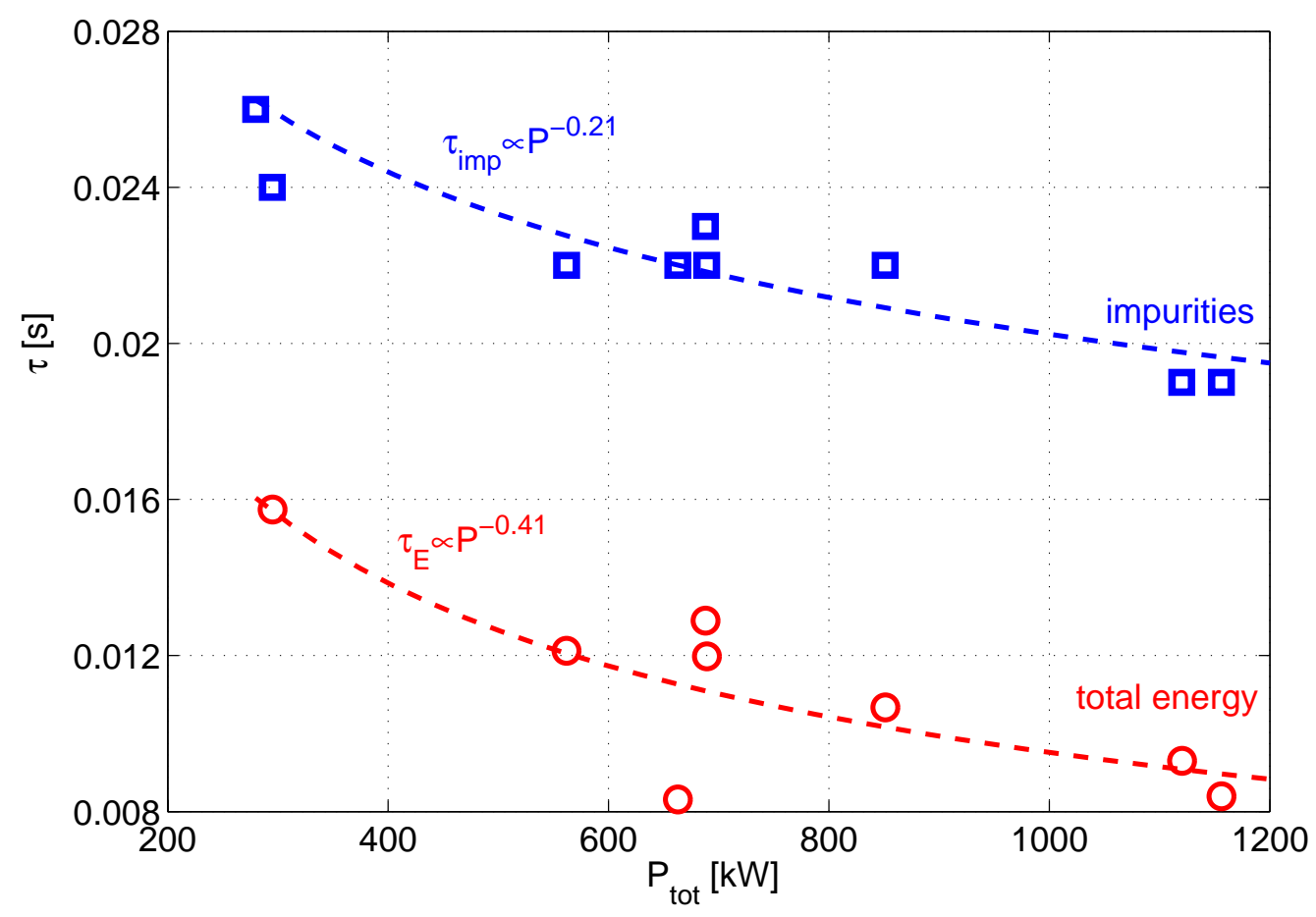

Fig.1 - Confinement time of injected impurities (squares) and plasma energy (circles) versus total power for $0 \leq P_{E C R H}<1 \mathrm{MW}$. The other parameters were $\kappa=1.6, \delta=0.33, \quad B_{T}=1.47 \mathrm{~T}, \quad I_{P}=260 \mathrm{kA}$, $\bar{n}_{e} \approx 1.5 \times 10^{19} \mathrm{~m}^{-3}$.

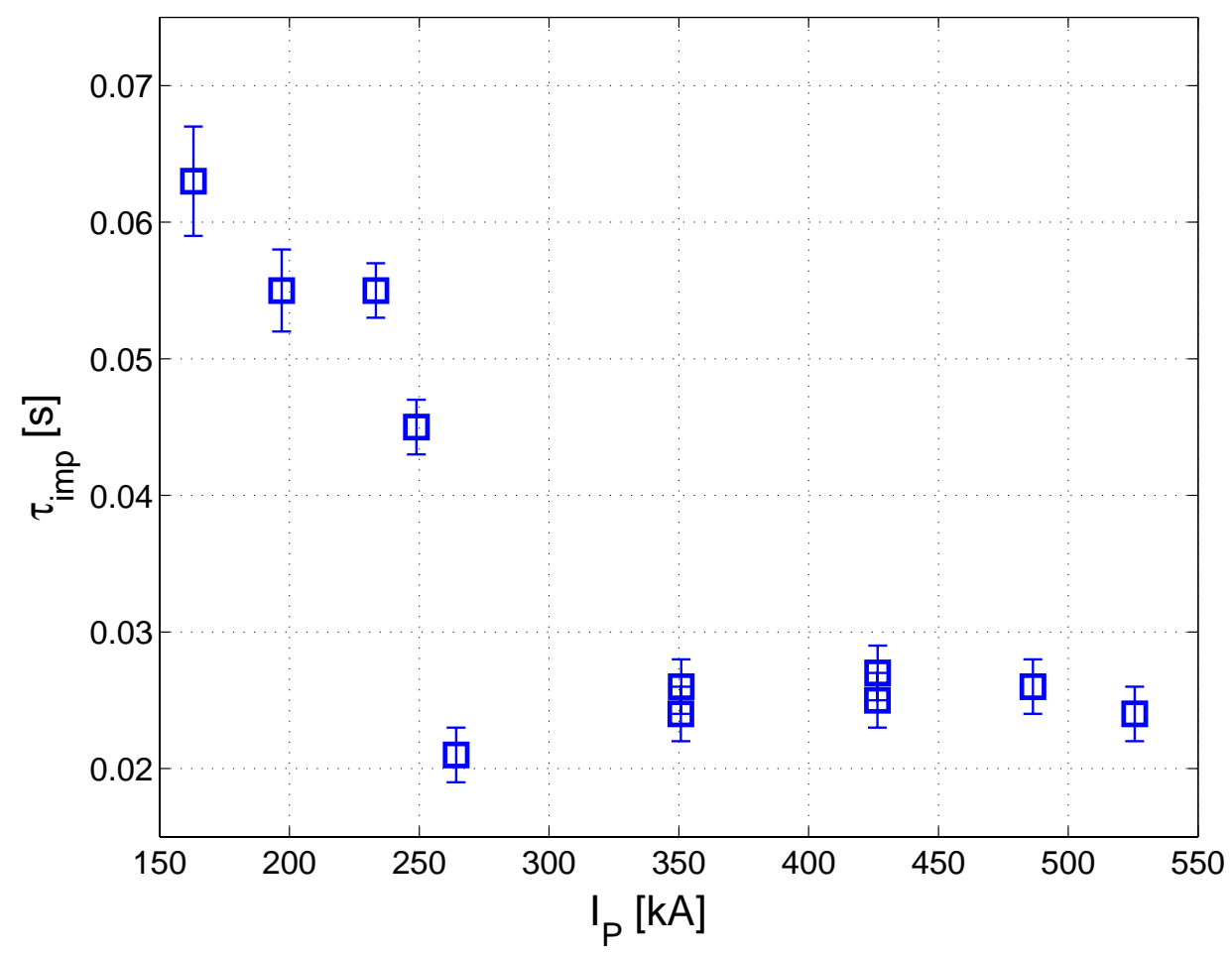

Fig.2 - Confinement time of injected impurities for different plasma currents, $163<I_{P}<525 \mathrm{kA}$. The other parameters were $\kappa=0.6, \delta=0.28, B_{T}=1.47 \mathrm{~T}, q_{95}=6.4 \div 2.2, \rho_{\text {inv }}=0.20 \div 0.55, \bar{n}_{e}=4 \times 10^{19} \mathrm{~m}^{-3}$. 



Fig.3 - Persistent impurity accumulation in a discharge with $I_{P}=235 \mathrm{kA}$. The line integrated SXR signals are shown, together with the position of the chords with respect to the plasma cross section in TCV, after sawtooth filtering by GSVD. SXR signals from central chords remain at a high level after penetration to the core, while SXR signals from more peripheral chords decrease back to the same background level as before the impurity injection.

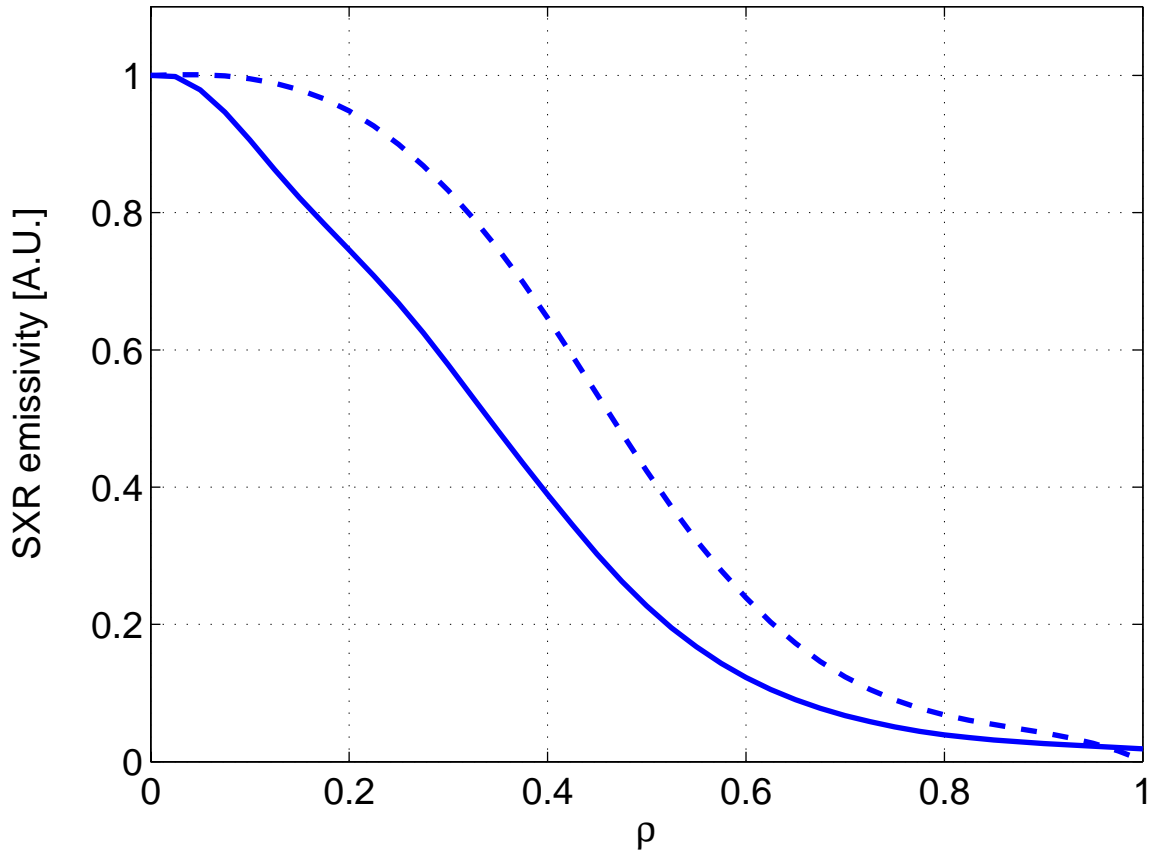

Fig.4 - Solid line: Normalised residual radial profile of the SXR emissivity caused by impurity radiation 200ms after injection into the discharge shown in fig.3. Broken line: Normalised profile obtained during the exponential decay phase at $I_{P}=350 \mathrm{kA}$ (no persistent accumulation). 


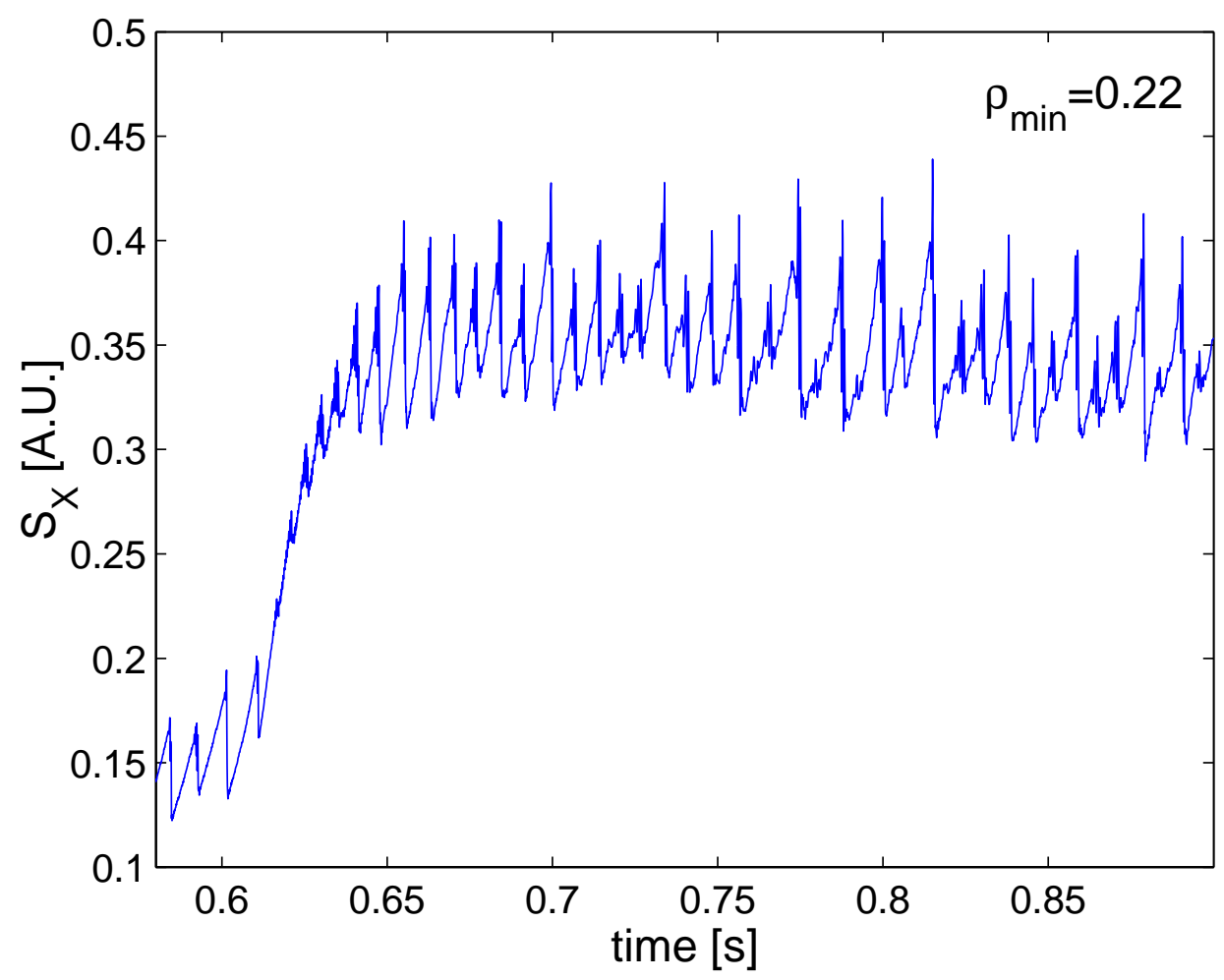

Fig.5 - The SXR signal from a central chord, not filtered, for the discharge with persistent impurity accumulation shown in fig.3. 